\title{
Editorial \\ Biomarkers: in combination they may do better
}

Frances MK Williams

Department of Twin Research and Genetic Epidemiology, King's College London, St Thomas' Hospital, Westminster Bridge Road, London SE1 7EH, UK

Corresponding author: Frances MK Williams, frances.williams@kcl.ac.uk

Published: 29 October 2009

Arthritis Research \& Therapy 2009, 11:130 (doi:10.1186/ar2839)

This article is online at http://arthritis-research.com/content/11/5/130

(C) 2009 BioMed Central Ltd

See related research by Dam et al., http://arthritis-research.com/content/11/4/R115

\begin{abstract}
The field of biomarkers is a growing one, particularly in osteoarthritis (OA). OA is the most common disabling condition in older persons and a major cause of morbidity. While the debate continues about which of the involved tissues - cartilage, bone or synovium - is the most important in OA aetiology, there is no doubt that the three develop abnormalities in concert; perhaps a truly useful biomarker will reflect just that. While efforts continue to identify reliable biomarkers useful for characterising the status, prognosis and measurement of treatment response in $\mathrm{OA}$, combining existing biomarkers to improve their accuracy looks promising.
\end{abstract}

Osteoarthritis (OA) is the most common joint condition in older persons and is among the leading causes of disability in the developed world. The study of biomarkers is a growing field, particularly in OA, a condition that finds itself pretty isolated among the diseases seen in rheumatology - with little in the way of treatment other than joint replacement surgery. A condition as common as $O A$ is in urgent need of disease-modifying treatment (DMOAD). As populations become heavier and more aged, $\mathrm{OA}$ is predicted to increase markedly in prevalence. The identification of biomarkers is one way to accelerate drug discovery and drug trials, the crucial rate-limiting step in bringing a novel therapeutic agent to the market. Biomarkers may be derived from all forms of biological variables, be they biochemical compounds, genetic loci or RNA fragments. Current biomarkers of interest in OA reflect the tissues involved in the pathology, from cartilage (for example, collagenous, type II collagen; and noncollagenous, cartilage oligomeric matrix protein) to bone (for example, collagenous, type I collagen; non-collagenous, osteocalcin) and synovium (for example, type III collagen).

Much biomarker research is, therefore, directed toward optimising the quantitation of changes in cartilage volume and turnover. Another advantage of combining biomarkers is the capture of information on both incidence of features and turnover of tissues. In the paper by Erik Dam and colleagues in the previous issue of Arthritis Research \& Therapy, the authors combined a biochemical marker with magnetic resonance imaging (MRI) features in an attempt to identify three important disease features: diagnosis, prognosis and efficacy of intervention [1]. MRI is used increasingly to document the various pathological features of $\mathrm{OA}$ and is unquestionably superior to plain radiographs. MRI allows the appreciation not only of joint space narrowing (representing cartilage loss) and osteophyte growth, but also of changes within bone (bone marrow lesions) and far greater capacity for assessing cartilage loss in minute detail by, for example, morphometric threedimensional analysis. Automated methods have been developed which quantify cartilage using this technique, although further validation is required. For an excellent review of biomarkers in OA, see Rousseau and Delmas [2].

Combining markers is intuitively the next step for a number of reasons and it is a route being adopted in a number of fields, not just in biomarkers; it makes good financial as well as scientific sense to use what has already been identified. No single biomarker will be a perfect descriptor of OA any more than one single pathological feature encapsulates the coordinated changes in the three tissues along with the cytokines released [3]. Biomarkers have a number of uses: in selecting patients for recruitment to drug trials, in early diagnosis, in prognosis and in measuring response to treatment. If bringing a new drug to market costs, say, $£ 10$ million (US $\$ 16.3$ million), then every month of a trial becomes crucial to the health economics of a pharmaceutical firm: if trials are too expensive, they won't be carried out and potential DMOADs will be lost. Because of the chronic and variable nature of $\mathrm{OA}$, new methods are needed to optimise the selection of patients for trials and as outcome measures in short, any route by which the long course of OA can be abbreviated into a more cost-effective time frame.

$\mathrm{DMOAD}=$ disease-modifying osteoarthritis drug; $\mathrm{KL}=$ Kellgren and Lawrence score; $\mathrm{MRI}=$ magnetic resonance imaging; $\mathrm{OA}=\mathrm{osteoarthritis}$. 
In the report of Dam and colleagues, subjects with normal health and with knee OA underwent baseline plain radiograph and $\mathrm{MRI}$, demographic data collection, blood and urine collection (for markers of cartilage turnover) and then repeat MRI at 21 months. The final analysis group comprised 287 knees scored conventionally using Kellgren and Lawrence as well as for knee size using the tibial plateau and MRI scans from a 0.18-Tesla extremity scanner that gave fully automated measures of volume, surface area and cartilage homogeneity. Biomarkers (image- and urine-derived) were judged by their ability at baseline to discriminate between cases and noncases $(K L>1$ versus $\mathrm{KL} \leq 1)$ and for prognostic value in order to separate the progressors from non-progressors. Receiveroperator curves of individual and combined biomarkers were generated. Results were expressed as odds ratio and area under the curve and so conformed to BIPED (burden of disease, investigative, prognostic, efficacy of intervention and diagnostic) recommendations [4].

As the authors comment, the use of the current suboptimal method for determining OA radiographically is a weakness and only early OA is included in the study. Results showed a $5 \%$ to $10 \%$ improvement in prediction using combined versus single biomarkers - a definite improvement on existing biomarkers. This is an important principle and should provide the stimulus for other groups to look in their biomarker collections and combine markers to optimise predictive value. DMOAD research over the next decade should be approached with optimism - millions of patients are waiting for something to alter the course of their disease.

\section{Competing interests}

The author declares that they have no competing interests.

\section{References}

1. Dam EB, Loog M, Christiansen C, Byrjalsen I, Folkesson J, Neilsen M, Qazi AA, Pettersen PC, Garnero P, Karsdal MA: Identification of progressors in osteoarthritis by combining biochemical and MRI-based markers. Arthritis Res Ther 2009, 11:R115.

2. Rousseau JC, Delmas PD: Biological markers in osteoarthritis. Nat Clin Pract Rheumatol 2007, 3:346-356.

3. Samuels J, Krasnokutsky S, Abramson SB: Osteoarthritis: a tale of three tissues. Bull NYU Hosp Jt Dis 2008, 66:244-250.

4. Bauer DC, Hunter DJ, Abramson SB, Attur M, Corr M, Felson D, Heinegard D, Jordan JM, Kepler TB, Lane NE, Saxne T, Tyree B, Kraus VB: Classification of osteoarthritis biomarkers: a proposed approach. Osteoarthritis Cartilage 2006, 14:723-727. 